\title{
Characterization of tumor response after administration of rituximab in pediatric B-NHL
}

\author{
Maria Bethke ${ }^{1}$, Georg Varga ${ }^{2}$, Toni Weinhage ${ }^{2}$, Harshana Sabharwal $^{1}$, Karin Mellgren ${ }^{3}$, \\ Gerrit Randau $^{1}$, Meike Rolfing ${ }^{1}$, Helmut Wittkowski ${ }^{2}$, Dirk Foell ${ }^{2}$, Ulf Michgehl ${ }^{1}$, and \\ Birgit Burkhardt ${ }^{1}$ \\ ${ }^{1}$ Pediatric Hematology and Oncology, University Children's Hospital Muenster \\ ${ }^{2}$ Department of Pediatric Rheumatology and Immunology, University Hospital Muenster \\ ${ }^{3}$ Sahlgrenska universitetssjukhuset
}

June 23, 2021

\begin{abstract}
Background Mature aggressive B-cell lymphoma are heterogenous malignancies that make up more than half of all diagnosed Non-Hodgkin lymphoma in children and adolescents. The overall survival rate increased over the last decades to 80-90\%, due to fine tuning of polychemotherapy. However, new therapeutic implications are needed to further increase the overall survival. Current clinical trials analyze the therapeutic effect of rituximab in pediatric patients, while the mechanism of action in vivo is still not fully understood. Methods Effector molecules important for tumor defense were analyzed before and at day five after rituximab treatment via flow cytometry. Serum rituximab levels were measured with an ELISA. Results We evaluated patient parameters that may affect treatment response in relation to rituximab administration and serum rituximab levels. We indeed found a reduction of FcrRII levels after rituximab treatment in monocyte subtypes, while FcrRI expression was significantly increased, pointing to exhaustion of FcrRII mediated B cell depletion and compensation via FcrRI mediated trogocytosis. Serum levels of proinflammatory marker proteins S100A8/A9 and S100A12 significantly decreased after treatment to normal levels from an overall proinflammatory state before treatment. CD57, perforin and granzyme B expression decreased after treatment, probably due to exhaustion of NK cells. Conclusion The highlighted effects of rituximab treatment on patient's immune response help understanding the biology behind tumor defense mechanisms and effector function. After subsequent studies, these novel insights might be translated into patient care and could contribute to improve treatment of pediatric patients with mature aggressive B-cell lymphoma.
\end{abstract}

\section{Introduction}

Mature aggressive B-cell lymphoma make up to 50 - $60 \%$ of all diagnosed Non-Hodgkin lymphoma (NHL) in children and adolescents in Germany. ${ }^{1}$ The most common subtypes of mature aggressive B-cell lymphoma are Burkitt lymphoma (BL), Burkitt leukemia (B-AL) and diffuse large B-cell lymphoma (DLBCL). ${ }^{2,3}$ Sporadic BL is less often associated with Epstein-Barr virus (EBV) infection compared to the endemic variant and is the most common variant in North America and Europe. ${ }^{3}$ In Germany, it is most frequent in children aged 414 and is primarily diagnosed in boys with a sex ratio of 4.5 to $1 .{ }^{4}$ With currently available polychemotherapy an overall survival rate of $80-90 \%$ can be reached. ${ }^{5,6}$ However, relapse of patients, which commonly occurs within six months after end of treatment, results in a very poor outcome and is fatal for most of the patients. ${ }^{4,7}$

The addition of the monoclonal antibody rituximab targeting CD20 on B cells contributed to the increased survival patients with advanced staged disease..$^{5,6,8,9}$ Although rituximab and its biosimilars are frequently applied in adults, there is still not much known about the mechanisms of action in vivo due to the lack of 
an adequate animal model. ${ }^{8}$ Additionally, biomarkers to identify patients responding to rituximab therapy are lacking. ${ }^{10}$ Therefore, better understanding of the malignancy and further development of therapeutic strategies are urgently needed.

Here, we show that expression of proteins important for tumor defense is altered at day five after rituximab treatment in patients suffering from B-NHL.

\section{Material and Methods}

\subsection{Patient samples and serum preparation}

All patients were recruited in the trial B-NHL 2013 (Eudra CT: 2013-003253-21) after informed consent (Table 1). NHL-BFM risk group stratification was performed as published earlier. According to protocol, treatment started with the rituximab window including one dose of rituximab $375 \mathrm{mg} / \mathrm{m}^{2}$ and one intrathecal triple drug administration. ${ }^{9}$ Samples were collected by the NHL-BFM study center as part of the trial BNHL 2013 prior to the start of treatment and at day five of treatment, prior to steroid treatment. Serum was generated using serum-separating tubes. To allow blood to clot, samples were left undisturbed at RT for $20 \mathrm{~min}$. To remove the clot, tubes were centrifuged for $10 \mathrm{~min}$ in a refrigerated centrifuge. The resulting supernatant was frozen until further use.

\subsection{Rituximab ELISA}

The rituximab ELISA is based on the methodology of Hampson et al., 2010. Briefly, a 96-well plate was coated with $100 \mu \mathrm{l} /$ well of rat $\alpha$-rituximab antibody (MB2A4, Bio-Rad, USA) and diluted 1:1000 in coating buffer overnight at $4^{\circ} \mathrm{C}$. After blocking for $2 \mathrm{~h}$ at RT with $200 \mu \mathrm{l} /$ well of $1 \%$ BSA in PBS, plates were washed three times with ELISA wash buffer. Samples were diluted 1:100000 in dilution buffer and Mabthera standard was prepared in dilution buffer at the following concentrations: $60,50,40,25,20,10,5,2.5$, $1,0 \mathrm{ng} / \mathrm{ml} .100 \mu \mathrm{l} /$ well of diluted sample or standard was then added to the plate and incubated for 60 min at RT. After washing the plate five times with ELISA wash buffer, HRP-conjugated goat $\alpha$-human IgG antibody (Merck, Germany) was diluted 1:60000 in blocking buffer and $100 \mu \mathrm{l} /$ well was added to the plate, followed by an incubation of 90 min at RT. The plate was washed again five times with ELISA wash buffer and $100 \mu \mathrm{l} /$ well of freshly prepared TMB HRP-substrate (BioLegend, USA) was added according to manufacturer's instructions. After incubation for $45 \mathrm{~min}$ in the dark, the reaction was terminated by 50 $\mu \mathrm{l} /$ well of $3 \mathrm{M}$ sulphuric acid. Absorbance was measured at $450 \mathrm{~nm}$ minus $570 \mathrm{~nm}$ for wavelength correction using a Synergy Mx Microplate Reader (Biotek, Germany). The standard curve was determined using a non-linear 4 parameter logistic regression (4PL) calibration model.

\subsection{Staining of cells and flow cytometry}

Flow cytometry measurements were performed on a CytoFLEX S flow cytometer (Beckman Coulter, Germany) and data were analyzed with the FlowJo software (version 10.0). For cell surface antigen staining, $5 \mu \mathrm{l}$ of each human TruStain FcX and True-Stain Monocyte Blocker (Biolegend, Germany) were added to $100 \mu \mathrm{l}$ of whole blood and incubated for $10 \mathrm{~min}$ at $4^{\circ} \mathrm{C}$. Surface staining was performed for $30 \mathrm{~min}$ at $4^{\circ} \mathrm{C}$ by directly adding fluorochrome-conjugated antibodies diluted 1:50 or 1:100 in PBS/1\% BSA. For lysis of the red blood cells, $2 \mathrm{ml}$ of 1x 1-step Fix/Lyse-solution (Thermo Fisher Scientific, Germany) was added to each tube and incubated for $40 \mathrm{~min}$ at RT. Subsequently, samples were washed with FACS buffer. $300 \mu \mathrm{l}$ of freshly prepared 1x Fix/Perm-buffer (Thermo Fisher Scientific, Germany) was then added to the cell pellet and incubated for 30 min at RT. After washing with 1x Perm-buffer (Thermo Fisher Scientific, Germany), intracellular staining was performed directly in the tube by adding $2 \mu$ of each antibody. After incubation for $30 \mathrm{~min}$ at RT, $500 \mu \mathrm{l}$ 1x Perm-buffer was added and samples were washed and treated with FACS buffer until measurement. The antibodies used for surface and intracellular staining are listed in Table S1.

\subsection{S100A8/A9 and S100A12 ELISA}

The S100A8/A9 ELISA was performed according to the manufacturers instructions (Bühlmann, Switzerland). For S100A12 serum level measurement, a 96 -well plate was coated with $50 \mu \mathrm{l} /$ well of rat $\alpha$-S100A12 
antibody (clone 11G7A1, Genescript biotech, USA) in coating buffer overnight at $4^{\circ} \mathrm{C}$. After blocking for $1 \mathrm{~h}$ at RT with $100 \mu \mathrm{l} /$ well of blocking buffer, plates were washed three times with ELISA wash buffer. Samples were diluted 1:4 in blocking buffer and S100A12 standard was prepared at the following concentrations: 16, $8,4,2,1,0.5,0.25$ and $0.125 \mathrm{ng} / \mathrm{ml}$. $50 \mu \mathrm{l} /$ well of diluted sample or standard was then added to the plate and incubated for $2 \mathrm{~h}$ at RT. After washing the plate three times with ELISA wash buffer, $50 \mu \mathrm{l} /$ well of biotinylated rat $\alpha$-S100A12 (clone 3G1D5-bio, Genescript biotech, USA) was added to the plate, followed by an incubation of $30 \mathrm{~min}$ at $37^{\circ} \mathrm{C}$. The plate was washed again three times with ELISA wash buffer and 50 $\mu \mathrm{l} /$ well of Streptavidin-peroxidase (Thermo Fisher Scientific, Germany) was added. After incubation for 30 min at $37^{\circ} \mathrm{C}$ and washing the plate three times, TMB substrate (Seramun Diagnostica GmbH, Germany) was added according to manufacturers instruction. After incubation for $10 \mathrm{~min}$ at RT in the dark, the reaction was terminated by $50 \mu \mathrm{l} /$ well of $2 \mathrm{~N} \mathrm{H}_{2} \mathrm{SO}_{4}$. Absorbance was measured at $450 \mathrm{~nm}$ using a Synergy $\mathrm{Mx}$ Microplate Reader (Biotek, Germany). The standard curve was determined using a non-linear 4 parameter logistic regression (4PL) calibration model.

\subsection{Statistical analysis}

Data are displayed in 10-90 percentile range with median. An unpaired two-tailed student's t-test was performed for independent sample comparisons using the GraphPad Prism software. For two-time point analysis a paired two-tailed student's t-test was performed. $P$-values [?]0.05 were considered significant with $p$ [?] $0.05(*), p$ [?] $0.01\left(^{* *}\right)$ and $p$ [?] $0.001\left(^{* * *}\right)$.

\section{Results}

3.1 Patients with limited disease have higher serum rituximab levels

To date the inter-individual variability of response of lymphoma patients to rituximab is still not understood. To better understand the turnover of rituximab, we monitored serum rituximab levels of 14 pediatric patients at day five after one dose of rituximab. The levels showed high inter-individual variety. Interestingly, there was a significant difference of the five-day rituximab levels between patients with limited disease compared to patients with advanced disease $(p=0.0001$, Fig. 1$)$. While patients with limited disease (risk groups R1 and R2) had a median level of $250 \mu \mathrm{g} / \mathrm{ml}$ rituximab, patients with advanced disease (risk groups R3 and R4) had a median five-day rituximab level of $40 \mu \mathrm{g} / \mathrm{ml}$.

$3.2 \mathrm{Fc} \gamma \mathrm{RI}$ and FcrRII expression on monocytes is altered upon rituximab treatment

$\mathrm{Fc} \gamma$ receptor $(\mathrm{Fc} \gamma \mathrm{R})$ mediated binding represents a relevant mechanism of action of rituximab induced $\mathrm{B}$ cell depletion in B-NHL. As linkers between malignant B cells and effector cells, FcrRs promote B cell depletion via antibody-dependent cellular cytotoxicity (ADCC), antibody-dependent phagocytosis (ADP) or trogocytosis. ${ }^{11}$ However, the importance of $\mathrm{Fc} \gamma \mathrm{R}$ mediated B cell depletion in vivo is still unknown. Therefore, $\mathrm{F} c \gamma \mathrm{R}$ expression was measured in monocyte subtypes of B-NHL cases $(\mathrm{n}=10)$ before and at day five after rituximab treatment. Monocyte subtypes can be distinguished by the expression of the glycoprotein CD14 and FcrRIII/CD16 on their cell surface. Vessel wall patrolling cells are called non-classical monocytes $\left(\mathrm{CD} 14^{\text {low }} \mathrm{CD} 16^{+}\right)$and migrating cells are called classical monocytes $\left(\mathrm{CD} 14^{+} \mathrm{CD} 16^{-}\right) \cdot{ }^{12} \mathrm{~A}$ third subset with a different expression profile was discovered later and is called intermediate monocytes $\left(\mathrm{CD} 14^{+} \mathrm{CD} 16{ }^{+}\right) \cdot{ }^{13}$ The subtypes are not only distinct in expression patterns, but also in function. ${ }^{14} \mathrm{Fc} \gamma \mathrm{RI}$ expression was significantly increased after rituximab treatment in classical monocytes $(p=0.002)$. While Fc $\gamma$ RIII expression was not altered, FcrRII expression was significantly decreased in classical and intermediate monocytes at day five after treatment ( $p=0.006$ and $p=0.023$, Fig. 2). Fc $\gamma \mathrm{R}$ expression was not altered in neutrophils $\left(\mathrm{CD}_{66} \mathrm{~b}^{+}\right)$and NK cells $\left(\mathrm{CD} 56^{+}\right)$(Supporting Information Fig. S1 and S2).

3.3 The expression of S100A9 and S100A12 in monocytes and neutrophils is increased after rituximab treatment while serum levels decreased

S100A9 and S100A12 are small calcium-binding proteins with proinflammatory properties that can trigger recruitment and activation of effector cells during early inflammatory events. They are highly expressed in the cytosol of neutrophils and monocytes during inflammation. ${ }^{15,16}$ To study the effect of rituximab on 
the early immune response and specifically on the activation of effector cells in B-NHL patients during treatment, the expression of S100A9 and S100A12 in monocyte subtypes was monitored before and at day five after rituximab treatment as shown by the flow cytometric analysis ( $\mathrm{n}=10$, Fig.3 A). S100A9 expression was significantly increased in classical, intermediate and non-classical monocytes after rituximab treatment ( $p=0.005, p=0.009$ and $p=0.031$, respectively). After rituximab exposure, S100A12 showed a significantly higher expression in classical and intermediate monocytes ( $p=0.037$ and $p=0.024$, respectively), whereby non-classical monocytes showed a similar tendency, although not significant. In addition, S100A9 and S100A12 expression in neutrophils was significantly increased in neutrophils at day five after rituximab treatment $(p=0.023$ and $p=0.008$, Supporting Information Fig. S3). Conversely, serum levels of S100A8/A9 and S100A12 significantly decreased after rituximab treatment $(\mathrm{n}=10$, Fig. $3 \mathrm{~B}, p=0.021$ and $p=0.012)$.

3.4 NK cell expression of CD57, perforin and granzyme B is decreased after rituximab treatment

NK cells are important mediators of ADCC and ADP and thereby highly contribute to rituximab mediated $\mathrm{B}$ cell depletion in patients suffering from B-NHL. ${ }^{17}$ However, their role during rituximab treatment in vivo is not fully understood. Therefore, we analyzed proteins involved in apoptotic and cytotoxic processes. The expression of CD57, perforin (PRF1) and Granzyme B (GrzB) was analyzed in NK cells before and at day five after rituximab treatment $(\mathrm{n}=12)$ in the B-NHL cohort. Surprisingly, CD57, PRF1 and GrzB expression were significantly decreased after rituximab treatment in NK cells $(p=0.006 ; p=0.045 ; p=$ 0.028, Fig. 4).

\section{Discussion}

The effectiveness of rituximab depends on several factors, including its activity and concentration in the environment of the tumor. To maximize therapeutic efficacy and to optimize dosing, a reliable assay for accurate measurement of rituximab levels in the bloodstream of patients is needed. ${ }^{18}$ Here, we show that serum rituximab levels vary highly among patients, who received standard doses of rituximab. In different studies, higher serum rituximab levels correlated with more favorable clinical response. ${ }^{19}$ In addition, initially measured tumor burden was reported to inversely correlate with serum rituximab levels. ${ }^{20}$ We show here that patients with limited disease (risk groups R1 and R2) have higher serum rituximab levels compared to patients of advanced disease (risk groups R3 and R4) at day five after rituximab administration. This correlates with previous findings of significantly better rituximab response in patients of risk group R3/R4. ${ }^{21}$ The advanced disease stage in R3/R4 patients translates into more available CD20 epitopes, where rituximab can attach. Consequently, less unbound rituximab molecules can be measured in the R3/R4 patient's serum. This rituximab ELISA is a reliable method to measure serum rituximab levels on a routinely basis in the future and can easily be implemented in daily routine.

ADCC, mediated by FcrRs of NK cells, is thought to be the main rituximab mechanism of action. ${ }^{22}$ Fc $\gamma$ RI expression on classical monocytes was shown to be increased at day five after rituximab treatment, while FcrRII expression significantly decreased. FcyRII can consist of two isoforms, FcyRIIa and FcyRIIb. ${ }^{23}$ The

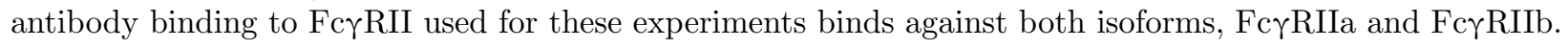
However, both isoforms have different functions in B cell depletion, FcrRIIa being activatory on target effector cells and FcrRIIb being inhibitory. ${ }^{23}$ It is tempting to speculate that monocytes expressing the activatory FcrRIIa might be exhausted at day five after rituximab treatment. In DLBCL patients, low FcrRIIa expression levels at diagnosis were described to lead to impaired rituximab-mediated cytotoxicity of NK cells. ${ }^{24}$ Also, expression of inhibitory Fc $\gamma$ RIIb might be reduced on the circulating effector cells after treatment since this Fc receptor type was described to negatively influence rituximab-mediated B cell depletion. ${ }^{23}$

$\mathrm{Fc} \gamma \mathrm{RI}$ is the main receptor that mediates trogocytosis, describing the process where effector cells remove rituximab-CD20 complexes, which can lead to rituximab resistance but also to target cell death. ${ }^{25}$ The mechanism was described to be less effective in rituximab mediated B cell depletion but is initiated when other mechanisms are exhausted. ${ }^{26}$ This supports the upregulated expression of Fc $\gamma R I$ at day five after treatment with downregulated FcrRII expression, due to exhaustion. 
Although being the main receptor mediating ADCC, expression of Fc $\gamma$ RIII remained unchanged in monocytes and NK cells. This might indicate that FcrRIII mediated B cell depletion via ADCC or ADP is not exhausted at day five after rituximab treatment and plays a role in an earlier time of the treatment. Fc $\mathrm{R}$ expression of neutrophils did not change, probably because neutrophils are not the predominant effector cells at day 5 after treatment. In neutrophils, only FcrRIIIb is expressed on the surface, rather mediating phagocytosis than ADCC. ${ }^{27}$

S100A9 and S100A12 belong to the S100 family and act via Toll-like receptors (TLRs) and receptors for advanced glycation end products (RAGEs). ${ }^{28-30}$ Expression of S100A9 is highest in monocytes and neutrophils, while S100A12 is mainly expressed by neutrophils. ${ }^{31,32}$ High serum levels of S100A8, S100A9 and S100A12 are associated with enhanced proinflammatory activity, predominantly found in patients with inflammatory or autoimmune diseases. ${ }^{33,34}$ Therefore, S100A9 and S100A12 can be used as valuable serum inflammatory markers. ${ }^{33,34}$ Furthermore, S100A9 could be used as a prognostic factor of survival in advanced ENKL patients. ${ }^{35}$ Additionally, a combination of serum S100A8/A9 and S100A12 was shown to predict rituximab treatment response in lupus nephritis. ${ }^{36}$ The expression of the proinflammatory proteins S100A9 and S100A12 was found to be significantly increased in monocytes and neutrophils at day five after rituximab treatment. Conversely, S100A8/A9 and S100A12 serum levels significantly decreased and therefore secretion by effector cells is lower at day five after treatment. Serum S100A8/A9 and S100A12 levels are similar to healthy individuals after rituximab treatment, concluding they are slightly increased before treatment indicating signs of inflammation. ${ }^{37,38}$ Increase in intracellular expression is accompanied with decrease in secretion of S100A9 and S100A12 by effector cells and vice versa, probably depicting the location of the proteins at the measured timepoint.

Among others, S100 proteins may, directly or indirectly, lead to recruitment and activation of NK cells that are the main effector cells that deplete B cells. It will be interesting to examine these mechanisms in future studies to point to new possibilities of activation and inhibition.

CD57+ cells express more perforin (PRF1) and granzyme B (GrzB), which are crucial for lytic activity of NK cells and are primed effectors for ADCC. ${ }^{39}$ Interestingly, expression of CD57, PRF1 and GrzB was significantly decreased at day five after rituximab administration. In other studies, high expression of CD57, PRF1 and GrzB correlated with highly active NK cells. ${ }^{39,40}$ Since GrzB and PRF1 both predominantly contribute to ADCC and we identified a decrease in expression, it is tempting to speculate that ADCC by NK cells might be the main mechanism of action in patients suffering from B-NHL. ${ }^{40}$ This could indicate that the NK cell population with high B cell depletion activity might be exhausted at day five after rapid NK cell recruitment in the first days after rituximab administration. By measuring the lytic activity of NK cells from patient samples, this effect could be studied further. However, the predominant NK cell population after rituximab treatment might be less mature since expression of GrzB and PRF1 directly correlates with cellular maturity. ${ }^{41}$ Additionally, different mechanisms of action may play a role at various timepoints. In future studies, it would be feasible to monitor the expression after administration of rituximab over time to improve rituximab therapy and to identify new diagnostic markers.

\section{Conflict of Interest statement}

The authors declare that there is no conflict of interest.

\section{Acknowledgements}

Thanks to the Kinderkrebshilfe Münster e.V. for funding parts of this study. Special thanks to the family of Joe Heckt, for their contribution and support. The clinical trial B-NHL 2013 is supported by the Deutsche Krebshilfe.

\section{References}

1. Burkhardt B, Zimmermann M, Oschlies I, et al. The impact of age and gender on biology, clinical features and treatment outcome of non-Hodgkin lymphoma in childhood and adolescence. Br J Haematol . 2005;131(1):39-49. 
2. Swerdlow SH, Campo E, Pileri SA, et al. The 2016 revision of the World Health Organization classification of lymphoid neoplasms. Blood . 2016;127(20):2375-2390.

3. Molyneux EM, Rochford R, Griffin B, et al. Burkitt's lymphoma.Lancet . 2012;379(9822):1234-1244.

4. Woessmann W, Zimmermann M, Meinhardt A, et al. Progressive or relapsed Burkitt lymphoma or leukemia in children and adolescents after BFM-type first-line therapy. Blood . 2020;135(14):1124-1132.

5. Woessmann W, Seidemann K, Mann G, et al. The impact of the methotrexate administration schedule and dose in the treatment of children and adolescents with B-cell neoplasms: a report of the BFM Group Study NHL-BFM95. Blood . 2005;105(3):948-958.

6. Minard-Colin V, Aupérin A, Pillon M, et al. Rituximab for High-Risk, Mature B-Cell Non-Hodgkin's Lymphoma in Children. N Engl J Med . 2020;382(23):2207-2219.

7. Burkhardt B, Taj M, Garnier N, et al. Treatment and Outcome Analysis of 639 Relapsed Non-Hodgkin Lymphomas in Children and Adolescents and Resulting Treatment Recommendations. Cancers (Basel) . $2021 ; 13(9)$.

8. Pishko A, Nasta SD. The role of novel immunotherapies in non-Hodgkin lymphoma. Transl Cancer Res . 2017;6(1):93-103.

9. Meinhardt A, Burkhardt B, Zimmermann M, et al. Phase II window study on rituximab in newly diagnosed pediatric mature B-cell non-Hodgkin's lymphoma and Burkitt leukemia. J Clin Oncol . 2010;28(19):31153121.

10. Hampson G, Ward TH, Cummings J, et al. Validation of an ELISA for the determination of rituximab pharmacokinetics in clinical trials subjects. J Immunol Methods . 2010;360(1-2):30-38.

11. Zhuang Y, Xu W, Shen Y, Li J. Fcr receptor polymorphisms and clinical efficacy of rituximab in nonHodgkin lymphoma and chronic lymphocytic leukemia. Clin Lymphoma Myeloma Leuk . 2010;10(5):347-352.

12. Kapellos TS, Bonaguro L, Gemünd I, et al. Human Monocyte Subsets and Phenotypes in Major Chronic Inflammatory Diseases. Front Immunol . 2019;10:2035.

13. Passlick B, Flieger D, Ziegler-Heitbrock HW. Identification and characterization of a novel monocyte subpopulation in human peripheral blood. Blood . 1989;74(7):2527-2534.

14. Zawada AM, Schneider JS, Michel AI, et al. DNA methylation profiling reveals differences in the 3 human monocyte subsets and identifies uremia to induce DNA methylation changes during differentiation. Epigenetics . 2016;11(4):259-272.

15. Foell D, Wittkowski H, Kessel C, et al. Proinflammatory S100A12 can activate human monocytes via Toll-like receptor 4. Am J Respir Crit Care Med . 2013;187(12):1324-1334.

16. Ryckman C, Vandal K, Rouleau P, Talbot M, Tessier PA. Proinflammatory activities of S100: proteins S100A8, S100A9, and S100A8/A9 induce neutrophil chemotaxis and adhesion. J Immunol . 2003;170(6):32333242 .

17. Merkt W, Lorenz HM, Watzl C. Rituximab induces phenotypical and functional changes of NK cells in a non-malignant experimental setting. Arthritis Res Ther . 2016;18(1):206.

18. Beum PV, Kennedy AD, Taylor RP. Three new assays for rituximab based on its immunological activity or antigenic properties: analyses of sera and plasmas of RTX-treated patients with chronic lymphocytic leukemia and other B cell lymphomas. J Immunol Methods . 2004;289(1-2):97-109.

19. Berinstein NL, Grillo-Lopez AJ, White CA, et al. Association of serum Rituximab (IDEC-C2B8) concentration and anti-tumor response in the treatment of recurrent low-grade or follicular non-Hodgkin's lymphoma.AnnOncol . 1998;9(9):995-1001. 
20. Illidge TM, Bayne M, Brown NS, et al. Phase 1/2 study of fractionated (131)I-rituximab in low-grade B-cell lymphoma: the effect of prior rituximab dosing and tumor burden on subsequent radioimmunotherapy. Blood . 2009;113(7):1412-1421.

21. Burkhardt B, Yavuz D, Zimmermann M, et al. Impact of Fc gamma-receptor polymorphisms on the response to rituximab treatment in children and adolescents with mature B cell lymphoma/leukemia. Ann Hematol . 2016;95(9):1503-1512.

22. Golay J, Introna M. Mechanism of action of therapeutic monoclonal antibodies: promises and pitfalls of in vitro and in vivo assays. Arch Biochem Biophys . 2012;526(2):146-153.

23. Lee CS, Ashton-Key M, Cogliatti S, et al. Expression of the inhibitory Fc gamma receptor IIB (FCGR2B, CD32B) on follicular lymphoma cells lowers the response rate to rituximab monotherapy (SAKK 35/98). $\mathrm{Br}$ J Haematol . 2015;168(1):145-148.

24. Danielou-Lazareth A, Henry G, Geromin D, et al. At diagnosis, diffuse large B-cell lymphoma patients show impaired rituximab-mediated NK-cell cytotoxicity. Eur J Immunol . 2013;43(5):1383-1388.

25. Velmurugan R, Challa DK, Ram S, Ober RJ, Ward ES. Macrophage-Mediated Trogocytosis Leads to Death of Antibody-Opsonized Tumor Cells. Mol Cancer Ther . 2016;15(8):1879-1889.

26. Beum PV, Peek EM, Lindorfer MA, et al. Loss of CD20 and bound CD20 antibody from opsonized $\mathrm{B}$ cells occurs more rapidly because of trogocytosis mediated by Fc receptor-expressing effector cells than direct internalization by the B cells. J Immunol . 2011;187(6):3438-3447.

27. Golay J, Da Roit F, Bologna L, et al. Glycoengineered CD20 antibody obinutuzumab activates neutrophils and mediates phagocytosis through CD16B more efficiently than rituximab. Blood . 2013;122(20):3482-3491.

28. Foell D, Frosch M, Sorg C, Roth J. Phagocyte-specific calcium-binding S100 proteins as clinical laboratory markers of inflammation. Clin Chim Acta . 2004;344(1-2):37-51.

29. Donato R, Cannon BR, Sorci G, et al. Functions of S100 proteins. Curr Mol Med . 2013;13(1):24-57.

30. Markowitz J, Carson WE, 3rd. Review of S100A9 biology and its role in cancer. Biochim Biophys Acta . 2013;1835(1):100-109.

31. Vogl T, Pröpper C, Hartmann M, et al. S100A12 is expressed exclusively by granulocytes and acts independently from MRP8 and MRP14.J Biol Chem . 1999;274(36):25291-25296.

32. Pruenster M, Vogl T, Roth J, Sperandio M. S100A8/A9: From basic science to clinical application. Pharmacol Ther . 2016;167:120-131.

33. Roth J, Goebeler M, Sorg C. S100A8 and S100A9 in inflammatory diseases. Lancet . 2001;357(9261):1041.

34. Meijer B, Gearry RB, Day AS. The role of S100A12 as a systemic marker of inflammation. Int J Inflam . 2012;2012:907078.

35. Zhou Z, Li Z, Sun Z, et al. S100A9 and ORM1 serve as predictors of therapeutic response and prognostic factors in advanced extranodal NK/T cell lymphoma patients treated with pegaspargase/gemcitabine. Sci Rep . 2016;6:23695.

36. Davies JC, Midgley A, Carlsson E, et al. Urine and serum S100A8/A9 and S100A12 associate with active lupus nephritis and may predict response to rituximab treatment. RMD Open . 2020;6(2).

37. Anink J, Van Suijlekom-Smit LW, Otten MH, et al. MRP8/14 serum levels as a predictor of response to starting and stopping anti-TNF treatment in juvenile idiopathic arthritis. Arthritis Res Ther . 2015;17(1):200.

38. Wittkowski H, Frosch M, Wulffraat N, et al. S100A12 is a novel molecular marker differentiating systemic-onset juvenile idiopathic arthritis from other causes of fever of unknown origin. Arthritis Rheum . 2008;58(12):3924-3931. 
39. Kared H, Martelli S, Ng TP, Pender SL, Larbi A. CD57 in human natural killer cells and T-lymphocytes. Cancer Immunol Immunother . 2016;65(4):441-452.

40. Smith MR. Rituximab (monoclonal anti-CD20 antibody): mechanisms of action and resistance. Oncogene . 2003;22(47):7359-7368.

41. Chattopadhyay PK, Betts MR, Price DA, et al. The cytolytic enzymes granyzme A, granzyme B, and perforin: expression patterns, cell distribution, and their relationship to cell maturity and bright CD57 expression. J Leukoc Biol . 2009;85(1):88-97.

\section{Legends}

Figure 1: Patients with limited disease show higher serum rituximab levels. Serum rituximab levels of the B-NHL cohort at day five after treatment. Rituximab levels $(\mu \mathrm{g} / \mathrm{ml})$ of patients, grouped after the B-NHL stratification criteria in R1/R2 $(\mathrm{n}=6)$ and R3/R4 $(\mathrm{n}=8)$, were measured with an ELISA at day five after treatment. Patients with limited disease (risk groups R1 and R2) presented with a significantly higher median serum rituximab level of $250 \mu \mathrm{g} / \mathrm{ml}$ than patients with advanced disease (risk groups R3 and R4) with $40 \mu \mathrm{g} / \mathrm{ml}(p=0.0001)$. An unpaired two-tailed student's t-test was performed.

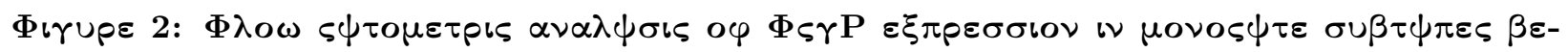

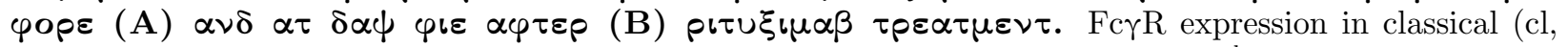
$\mathrm{CD} 14^{+} \mathrm{CD} 16^{-}$), intermediate (int, $\mathrm{CD} 14^{+} \mathrm{CD} 16^{+}$) and non-classical (non-cl, CD14 ${ }^{\text {low }} \mathrm{CD} 16^{+}$) monocytes was analyzed. Fc $\gamma$ RI expression was significantly increased in classical monocytes $(p=0.002)$. FcrRII expression was significantly decreased in classical and intermediate monocytes, while FcrRIII expression was not influenced by rituximab treatment $(p=0.023$ and $p=0.006)$. Samples were measured in duplicates $(\mathrm{n}$ $=10$ ). Expression levels are displayed as median fluorescent intensity (MFI). A paired two-tailed student's t-test was performed.

Figure 3: S100A9 and S100A12 expression is increased in monocyte subtypes at day five after rituximab treatment while serum levels are decreased. A S100A9 and S100A12 expression was measured via flow cytometry before (A) and at day five after (B) rituximab treatment in classical (cl, $\mathrm{CD} 14^{+} \mathrm{CD} 16^{-}$), intermediate (int, $\mathrm{CD} 14^{+} \mathrm{CD} 16^{+}$) and non-classical (non-cl, CD14 ${ }^{\text {low }} \mathrm{CD} 16^{+}$) monocytes. S100A9 expression significantly increased after rituximab treatment in all monocyte subtypes $(p=0.005, p$ $=0.009$ and $p=0.031)$. S100A12 expression significantly increased in classical and intermediate monocytes after rituximab treatment $(p=0.037$ and $p=0.024)$. Expression levels are displayed as median fluorescent intensity (MFI). A paired two-tailed student's t-test was performed $(\mathrm{n}=10)$. B Serum S100A8/9 and S100A12 levels were measured with an ELISA before (A) and at day five after (B) rituximab treatment. Conversely to expression levels, serum S100A8/9 and S100A12 levels significantly decreased after treatment $(p=0.021$ and $p=0.012)$. Values are displayed in $\mathrm{ng} / \mathrm{ml}$. A paired two-tailed student's t-test was performed $(\mathrm{n}=10)$.

Figure 4: CD57, PRF1 and GrzB expression is decreased in NK cells at day 5 after rituximab treatment. CD57, perforin (PRF1) and granzyme B (GrzB) expression was measured via flow cytometry before (A) and at day five after (B) rituximab treatment in NK cells. CD57 ( $p=0.006)$, PRF1 $(p=0.045)$ and GrzB $(p=0.028)$ expression were significantly decreased at day five after rituximab treatment in NK cells. Expression levels are displayed as median fluorescent intensity (MFI) in CD56 ${ }^{+}$positive cells. A paired two-tailed student's t-test was performed $(\mathrm{n}=12)$.

Table 1: Clinical characteristics of B-NHL patients.

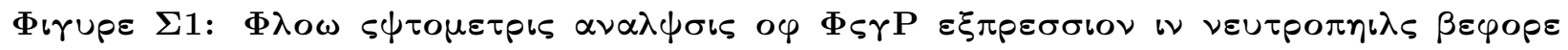

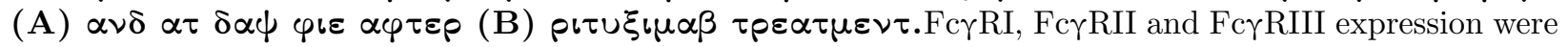
not altered by rituximab treatment in neutrophils $(\mathrm{n}=15)$. Expression levels are displayed as median fluorescent intensity (MFI) in CD66 $\mathrm{b}^{+}$positive cells. A paired two-tailed student's t-test was performed.

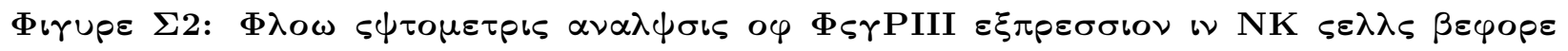




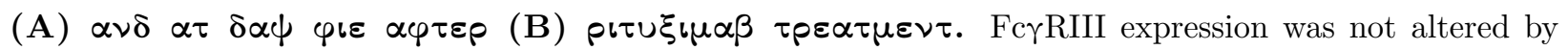
rituximab treatment in NK cells $(\mathrm{n}=12)$. Expression levels are displayed as median fluorescent intensity (MFI) in CD56 ${ }^{+}$positive cells. A paired two-tailed student's t-test was performed.

Figure S3: S100A9 and S100A12 expression in neutrophils was increased after rituximab treatment. Flow cytometric analysis showed an increase in expression of S100A9 and S100A12 in neutrophils at day five after rituximab treatment (B) compared to the start of the treatment $(\mathrm{A})(p=0.023$ and $p=$ 0.008). Expression levels are displayed as median fluorescent intensity (MFI) in CD66 b positive cells. A paired two-tailed student's t-test was performed $(\mathrm{n}=15)$.

Table S1: Fluorochrome-conjugated antibodies used for flow cytometry analyses.

\section{Serum rituximab levels}

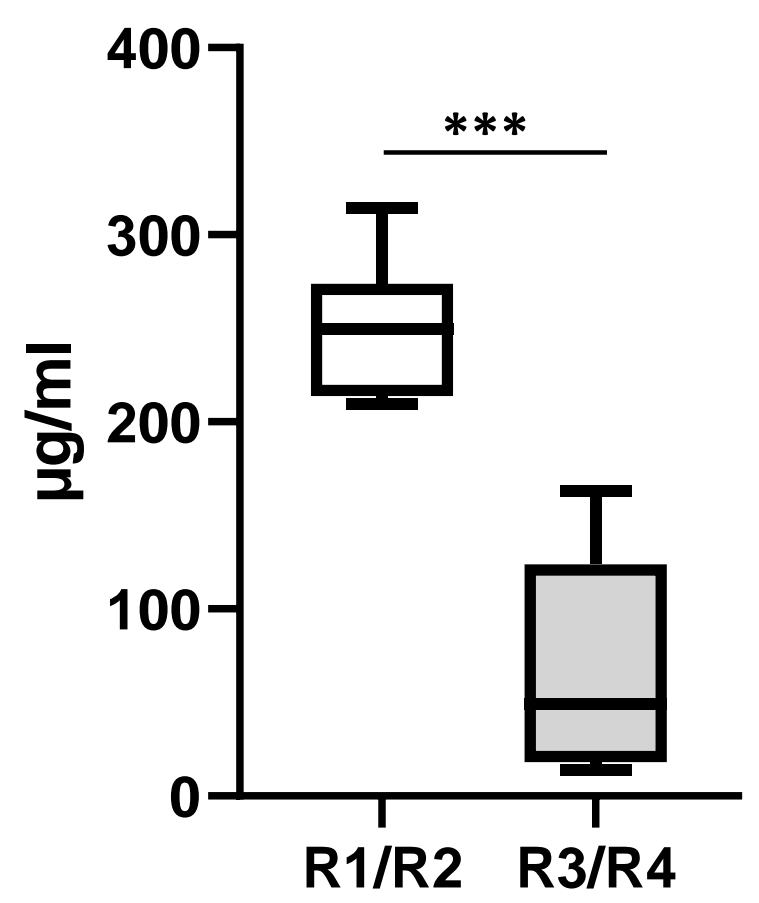




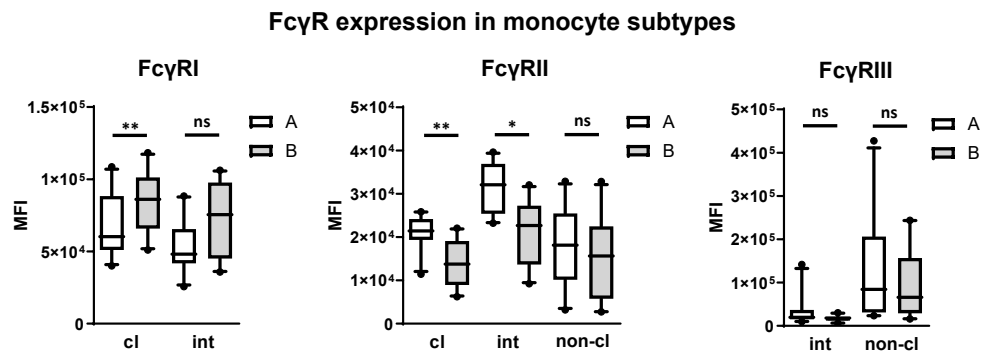


Expression in monocyte subtypes and serum level of $\mathbf{S} 100$
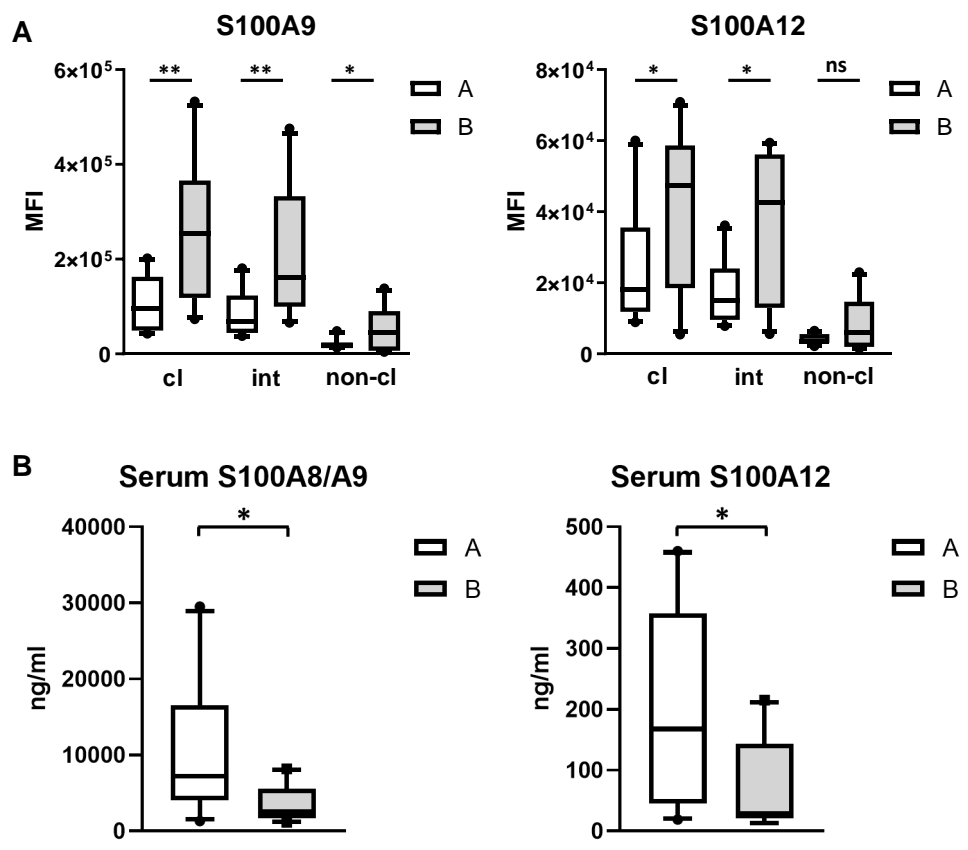


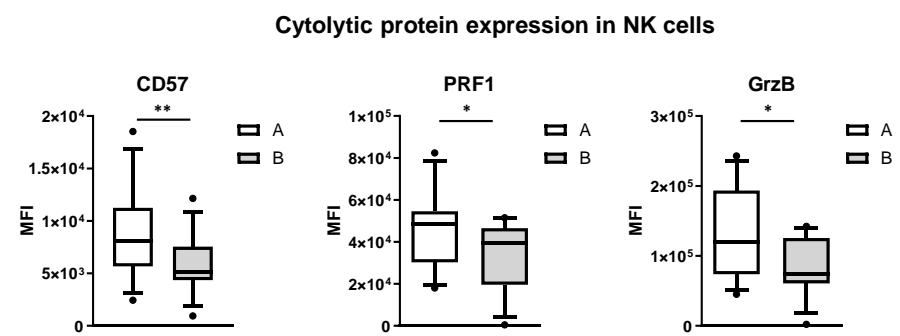

\section{Hosted file}

Table 1.docx available at https://authorea.com/users/421413/articles/527459-characterizationof-tumor-response-after-administration-of-rituximab-in-pediatric-b-nhl 